\title{
Comment la bio-cristallographie permet de proposer un mécanisme d'action des gaz anesthésiques
}

Nathalie Colloc'h' ${ }^{(1)}$ (colloch@cyceron.fr), Jacques H. Abraini ${ }^{(1)}$ et Thierry Prangé(2)

(1) CI-NAPS, UMR 6232 - Université de Caen - CNRS, Centre Cyceron, 14074 Caen Cedex 5

(2) LCRB, UMR 8015 - Université Paris Descartes - CNRS, Faculté de Pharmacie, 75270 Paris Cedex 06

Les gaz inertes sont utilisés pour leurs propriétés anesthésiques. Récemment, la découverte de leurs propriétés neuroprotectrices a relancé les recherches sur les mécanismes moléculaires mis en jeu, encore mal connus.

Nous avons déterminé,

sous pression de xénon

ou de protoxyde d'azote,

la structure de l'urate oxydase

et de I'annexine V, deux

protéines modèles de celles impliquées dans l'anesthésie.

Cette étude a montré que ces deux gaz occupaient le même site de liaison principal.

Les relations observées entre l'expansion du volume des cavités de liaison des gaz et leurs pouvoirs anesthésiants ont permis de proposer un mécanisme d'action en deux étapes.

Les termes suivis d'un astérisque $(\star)$ sont définis dans le glossaire, p. 13.

\section{Mécanismes d'action des gaz anesthésiques}

Les gaz inertes ou, plus exactement, métaboliquement inertes que sont le protoxyde d'azote $\left(\mathrm{N}_{2} \mathrm{O}\right)$ et le xénon, sont utilisés depuis plusieurs années dans la pratique médicale pour leurs propriétés narcotiques, anesthésiques et analgésiques. Les propriétés analgésiques et sédatives du protoxyde d'azote sont connues depuis le début du XIX ${ }^{\mathrm{e}}$ siècle [1]. Le xénon a commencé à être employé comme anesthésique en 1951 ; actuellement, son utilisation se développe (voir encadré, p.11). En plus de leur pouvoir anesthésiant similaire, le xénon et le protoxyde d'azote ont une action protectrice analogue sur la mort neuronale ischémique : ils limitent les effets délétères dus à un accident vasculaire cérébral [2].

Ce n'est que très récemment que les mécanismes à l'origine des effets sédatifs de ces deux gaz ont été partiellement identifiés. Après avoir longtemps pensé qu'ils produisaient leurs effets en dénaturant les lipides des membranes cellulaires, on sait maintenant qu'ils peuvent perturber directement les fonctions protéines des récepteurs à canal neuronal` inclus dans ces lipides [3]. Mais, au contraire de la plupart des anesthésiques volatils tel que le fluothane (halothane), qui agissent en augmentant l'activité du principal récepteur de la neurotransmission inhibitrice du système nerveux central, le $\mathrm{GABA}_{\mathrm{A}}{ }^{\star}$, le protoxyde d'azote et le xénon produisent surtout leurs effets en réduisant l'activité du principal récepteur de la neurotransmission excitatrice, le NMDA^, une protéine membranaire (voir la figure, p. 13) [4,5].

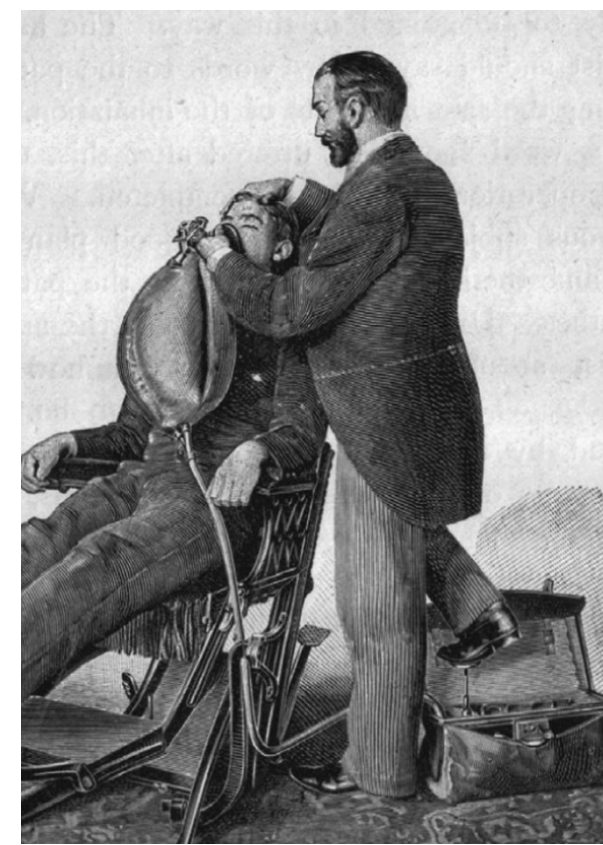

Anesthésiste administrant le mélange de protoxyde d'azote-oxygène. On remarquera que l'opérateur actionne la sortie des gaz avec son pied. (D'après F.W. Hewitt, The administration of nitrous oxide and oxygen for dental operations, C. Ash \& sons, Londres, 1897, p. 44). Courtoisie : Marguerite Zimmer

Les gaz inertes se fixent également sur un grand nombre de protéines globulaires, principalement dans des cavités internes hydrophobes [6].

Dans cette étude, nous avons cherché à préciser les sites et les mécanismes de liaison du protoxyde d'azote et du xénon sur leurs cibles biologiques.

Le xénon et le protoxyde d'azote ont-ils les mêmes sites de liaison?

Le xénon (Xe) est un gaz noble qui possède 54 électrons, et dont la masse molaire est de $131 \mathrm{~g} / \mathrm{mol}$; son rayon de van der Waals est de 2,24 A. Le protoxyde 
2. Vue en ruban de la chaîne protéique de l'annexine $V$, avec ses quatre domaines de structures similaires. À gauche (A), l'annexine V est dans sa conformation à basse concentration de calcium. À droite (B), elle est dans sa conformation à haute concentration de calcium : le glutamate 226 a basculé pour former le site de accessible au solvant ; l'atome de xénon ou une molécule de protoxyde d'azote se fixe au même endroit dans la cavité hydrophobe au centre du domaine III, laissée vacante par le mouvement du tryptophane 185. Une deuxième molécule de protoxyde d'azote se fixe au centre du domaine I. Le xénon, les protoxydes d'azote et les ions calcium sont représentés en modèle atomique, avec les azotes en bleu, les oxygènes en rouge, le xénon en orange, et les ions calcium en rose. liaison du calcium, et le tryptophane 185 est

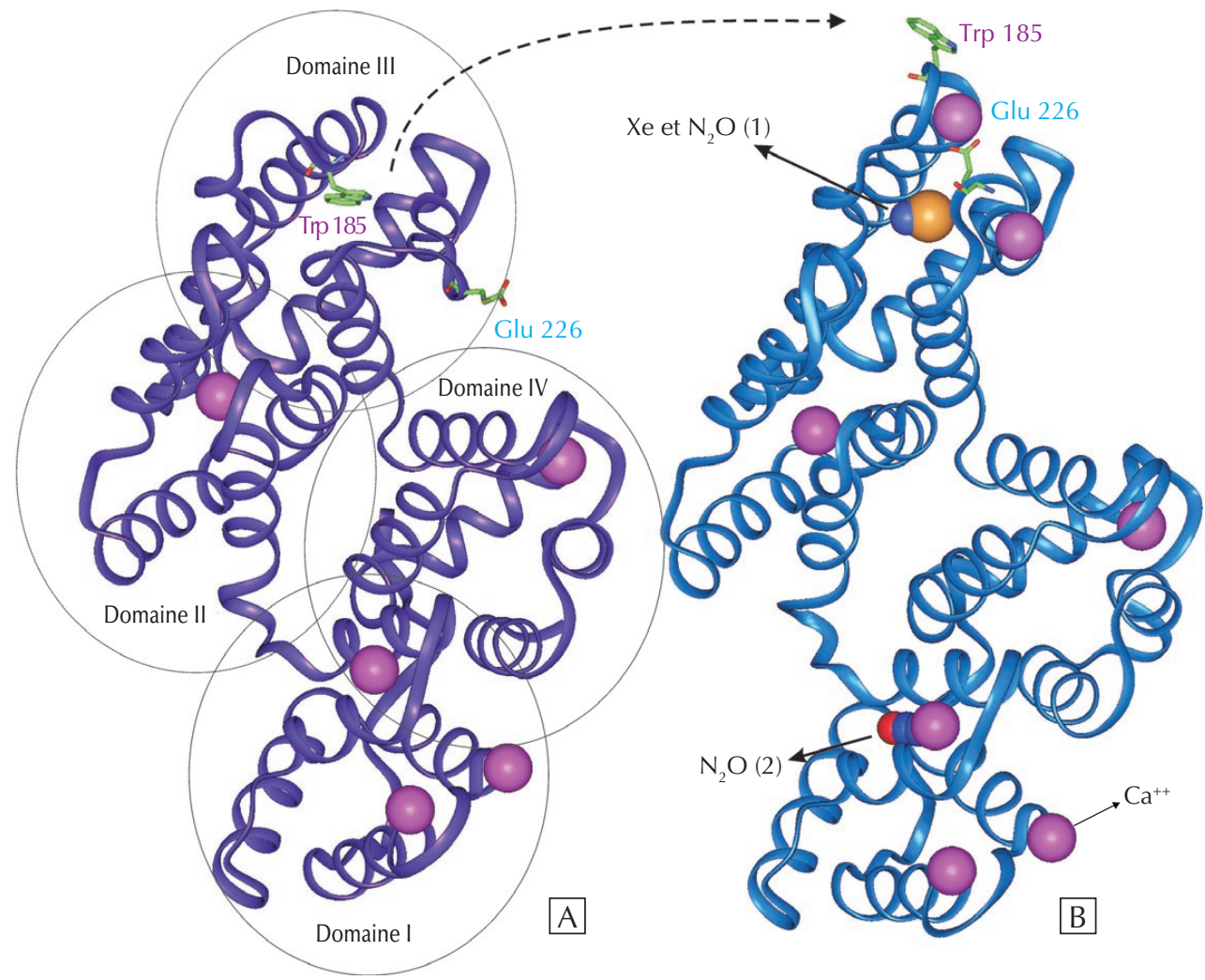

thésie. Ceci explique vraisemblablement la similitude des propriétés neuroprotectrices et anesthésiques de ces deux gaz.

de l'annexine $V$ en complexe avec le xénon ou le protoxyde d'azote

Aucune structure de complexe entre l'annexine $\mathrm{V}$ et un gaz n'avait été résolue auparavant : on ne savait donc pas si cette protéine possédait un site capable de lier un gaz. L'annexine $\mathrm{V}$ est composée de quatre domaines` structuralement similaires, chaque domaine possédant un site de liaison principal du calcium (fig. 2A). Son étude cristallographique sous pression de xénon ou de protoxyde d'azote a montré que ces gaz se fixent au même endroit, dans une cavité hydrophobe enfouie au sein du domaine III (fig. 2B). De plus, une seconde molécule de $\mathrm{N}_{2} \mathrm{O}$, moins présente que la première, se loge dans une cavité hydrophobe plus petite, localisée au sein du domaine I.

\section{Conclusion des études structurales}

Ces études par bio-cristallographie sous pression de gaz montrent que le site principal de liaison du xénon et du protoxyde d'azote est identique dans chacune des deux protéines modèles, suggérant que ces deux gaz ont des propriétés d'hydrophobie similaires.

Par analogie, nous faisons l'hypothèse qu'il en est de même dans les protéines qui sont physiologiquement impliquées dans l'anes-

\section{Implication de la liaison des gaz sur la fonction des protéines}

\section{Urate oxydase}

Nous avons utilisé l'urate oxydase comme prototype de protéine globulaire pouvant fixer un atome de gaz inerte dans une cavité, et dont le fonctionnement enzymatique pourrait être inhibé par cette présence. Cette inhibition a depuis été vérifiée expérimentalement. L'urate oxydase, elle même, est absente chez l'homme. Mais d'autres protéines globulaires intracellulaires possédant des cavités internes analogues, pourraient être impliquées dans les mécanismes de la neuroprotection et de l'anesthésie.

\section{Annexine V}

L'élévation de la concentration de calcium induit un important changement de conformation de l'annexine V (fig. 2). À basse concentration de calcium, le centre du domaine III est occupé par un résidu tryptophane ${ }^{\star}$ (Trp 185). À concentration de calcium plus élevée, un résidu glutamate* (Glu 226) bascule pour former le site de liaison du calcium, ce basculement entraînant un mouvement de la boucle portant le résidu tryptophane ; celui-ci se retrouve complètement accessible au solvant et prêt à se lier à la membrane lipidique. Au centre du domaine III, à la place du résidu tryptophane, se trouve maintenant une cavité hydrophobe. C'est dans cette cavité que le xénon ou le protoxyde d'azote se fixe (fig. 2B). Nous suggérons donc que la présence d'un gaz dans la cavité empêcherait, ou du moins perturberait, cet important changement de conformation indispensable au processus d'attachement et détachement de l'annexine $\mathrm{V}$ aux membranes, entraînant des modifications $\mathrm{du}$ fonctionnement cellulaire. Cette hypothèse peut être étendue aux récepteurs neuronaux NMDA : la présence d'un gaz dans une cavité proche du canal neuronal` par lequel passe l'ion calcium pourrait entraîner des perturbations importantes de ces récepteurs.

\section{Implication pour les mécanismes de l'anesthésie}

\section{Les deux étapes de l'anesthésie}

Suivant la concentration de gaz anesthésique employée, les effets obtenus ne sont pas les mêmes. Ainsi, une faible concentration 
de gaz entraîne des phénomènes d'hypnose et des troubles psychiques correspondant aux premières étapes de l'anesthésie. L'anesthésie dite chirurgicale, à savoir l'endormissement et l'absence de réponse à des stimuli de douleur, requiert des concentrations de gaz plus importantes. Pour comparer les gaz anesthésiques entre eux, leur pouvoir anesthésiant est évalué par leur mac: "minimum alveolar anesthetic concentration", la concentration minimale de gaz nécessaire pour atteindre un état donné. La mac-awake correspond à la concentration entraînant des phénomènes d'hypnose ou des troubles psychiques ; la mac-immobility correspond à celle nécessaire pour atteindre l'anesthésie chirurgicale.

Le rapport entre les mac-awake du protoxyde d'azote et du xénon est de 1,3 : ce dernier est donc 1,3 fois plus puissant que le $\mathrm{N}_{2} \mathrm{O}$ pour induire une hypnose et des troubles psychiques. Par contre, le rapport entre les mac-immobility des deux gaz est de l'ordre de 1 : ils sont donc équivalents pour induire une anesthésie chirurgicale.

\section{Relation entre 1'expansion de volume induite par les gaz et leur pouvoir anesthésiant}

Une théorie ancienne prédisait que le pouvoir anesthésiant des gaz était lié à l'expansion générée par leur présence. Nous avons donc calculé l'expansion du volume de la cavité de liaison des gaz dans les deux protéines modèles utilisées.

Dans l'urate oxydase, le volume de la cavité hydrophobe augmente en présence de gaz, et s'accroît 1,3 fois plus en présence de xénon qu'en présence de $\mathrm{N}_{2} \mathrm{O}$. $\mathrm{Ce}$ rapport d'expansion est similaire au rapport des pouvoirs anesthésiants des gaz évalués par leur mac-awake (les premières étapes de l'anesthésie).

Dans l'annexine V, le volume des cavités hydrophobes augmente en présence de $\mathrm{Xe}$ ou de $\mathrm{N}_{2} \mathrm{O}$, mais nous n'avons trouvé aucune relation entre le rapport des expansions et celui des pouvoirs anesthésiants. Par contre, si l'on additionne les volumes des cavités accessibles au xénon ou au protoxyde d'azote dans l'urate oxydase et dans l'annexine $\mathrm{V}$, comme un modèle d'occupation simultanée par les gaz de protéines globulaires et de récepteurs neuronaux, l'expansion globale des cavités est similaire en présence des deux gaz. Le rapport des expansions globales induites par $\mathrm{Xe}$ et $\mathrm{N}_{2} \mathrm{O}$ est donc de l'ordre de 1 , voisin du rapport de leurs pouvoirs anesthésiants évalués par leur mac-immobility (anesthésie chirurgicale).

\section{Le modèle proposé}

Ces deux relations observées entre l'expansion de volume induite par les gaz et leur pouvoir anesthésiant nous ont permis de proposer un modèle d'action des gaz anesthésiques en deux étapes :

- à faible concentration, ils se lieraient aux protéines globulaires possédant de larges cavités hydrophobes, cibles d'accès facile. La liaison des gaz modifierait la fonction de leurs protéines cibles, entraînant des perturbations cellulaires conduisant aux premières étapes de l'anesthésie, comme l'amnésie ou les troubles psychiques ;

- à plus haute concentration, quand tous les sites dans les protéines globulaires sont saturés, le gaz se lierait dans les cavités plus proches des canaux neuronaux, perturbant leur fonctionnement, ce qui conduirait à l'anesthésie chirurgicale (endormissement, absence de réponse à la douleur) [8].

\section{Références}

1 - M. Zimmer, Histoire de l'anesthésie - Méthodes et techniques au XIXe siècle, EDP Sciences, Les Ulis (2008)

2 • H.N. David et al., J. Cereb. Blood Flow Metab. 23 (2003) 1168-1173.

3 • N.P. Franks et W.R. Lieb, Nature 310 (1984) 559-601.

4 • N.P. Franks et al., Nature 396 (1998) 324.

5 • V. Jevtovic-Todorovic et al., Nat. Med. 4 (1998) 460-463.

6 • T. Prangé et al., Proteins 30 (1998) 61-73.

7 • M. Schiltz, T. Prangé, et R. Fourme, J. Appl. Cryst. 27 (1994) 950-960.

8 • N. Colloc'h et al., Biophys J. 92 (2007) 217-224.

\section{Glossaire}

Canal neuronal : Iorsque le neurotransmetteur, comme par exemple le glutamate ou le GABA (acide gamma-aminobutyrique), se fixe sur son récepteur, ce dernier change de forme et permet ainsi à des ions de traverser son canal central.

Domaine structural : partie d'une protéine qui a une conformation indépendante du reste de la chaîne. Glutamate, tryptophane : acides aminés naturels, composants des chaînes de protéines.

Protéine globulaire : protéine sans attache à la membrane, elle peut être extracellulaire ou intracellulaire. Les enzymes appartiennent majoritairement à cette catégorie de protéine.

Protéine membranaire : protéine se liant à la membrane, avec un ou plusieurs domaines transmembranaires, ou intégralement membranaire. Les récepteurs canaux sont des protéines membranaires.

Récepteur $\mathrm{GABA}_{\mathrm{A}}$ : récepteur canal neuronal de type $\mathrm{A}$ du GABA. Le GABA est le principal neurotransmetteur dans le cerveau des vertébrés.

Récepteur NMDA : récepteur canal neuronal du N-Methyl-D-Aspartate (NMDA), très présent dans les neurones, et impliqué dans la neurotransmission par le glutamate (voir figure ci-contre).

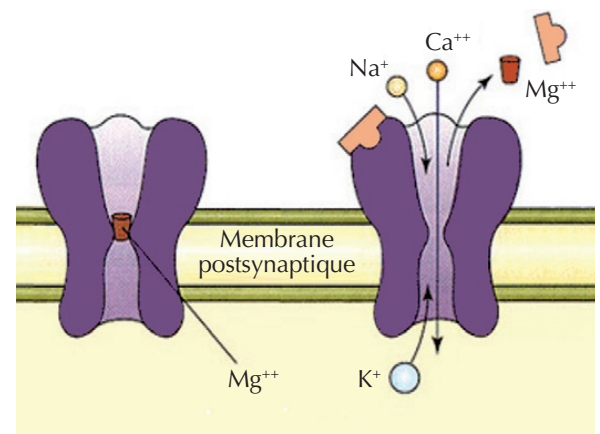

Fonctionnement du récepteur NMDA.

À gauche : le récepteur NMDA est bloqué par un ion $\mathrm{Mg}^{++}$ qui occupe le canal central ; le $\mathrm{Ca}^{++}$ne peut pas entrer. A droite : Iorsque des molécules de glutamate (accompagnées d'une dépolarisation électrique) se fixent sur le récepteur, ce dernier se déforme, l'ion $\mathrm{Mg}^{++}$est libéré, ce qui permet aux ions $\mathrm{Na}^{+}, \mathrm{K}^{+}$et $\mathrm{Ca}^{++}$de traverser le canal. l'entrée de calcium dans le neurone modifie l'intensité de la force de transmission synaptique. 